University of Nebraska - Lincoln

DigitalCommons@University of Nebraska - Lincoln

August 2000

\title{
Magnetic localization in transition-metal nanowires
}

\author{
Ralph Skomski \\ University of Nebraska-Lincoln, rskomski2@unl.edu \\ H. Zeng \\ University of Nebraska - Lincoln \\ M. Zheng \\ University of Nebraska - Lincoln \\ David J. Sellmyer \\ University of Nebraska-Lincoln, dsellmyer@unl.edu
}

Follow this and additional works at: https://digitalcommons.unl.edu/physicssellmyer

Part of the Physics Commons

Skomski, Ralph; Zeng, H.; Zheng, M.; and Sellmyer, David J., "Magnetic localization in transition-metal nanowires" (2000). David Sellmyer Publications. 58.

https://digitalcommons.unl.edu/physicssellmyer/58

This Article is brought to you for free and open access by the Research Papers in Physics and Astronomy at DigitalCommons@University of Nebraska - Lincoln. It has been accepted for inclusion in David Sellmyer Publications by an authorized administrator of DigitalCommons@University of Nebraska - Lincoln. 


\title{
Magnetic localization in transition-metal nanowires
}

\author{
R. Skomski, ${ }^{*}$ H. Zeng, M. Zheng, and D. J. Sellmyer \\ Department of Physics and Astronomy and Center for Materials Research and Analysis, University of Nebraska, Lincoln, Nebraska 68588
}

(Received 7 December 1999)

\begin{abstract}
Magnetization reversal in transition-metal nanowires is investigated. Model calculations explain why magnetization reversal is localized, as opposed to the sometimes assumed delocalized coherent-rotation and curling modes. The localization is a quite general phenomenon caused by morphological inhomogenities and occurring in both polycrystalline and single-crystalline wires. In the polycrystalline limit, the competition between interatomic exchange and anisotropy gives rise to a variety of random-anisotropy effects, whereas nearly single-crystalline wires exhibit a weak localization of the nucleation mode. Model predictions are used to explain the coercive and magnetic-viscosity behavior of $\mathrm{Co}$ (and $\mathrm{Ni}$ ) nanowires electrodeposited in selfassembled alumina pores.
\end{abstract}

\section{INTRODUCTION}

Since Ising's seminal proof that short-range interactions are unable to create ferromagnetism in spin chains, ${ }^{1}$ onedimensional magnets have attracted much attention. ${ }^{2-9} \mathrm{~A}$ fairly recent research area involves magnetic nanowires, which are of potential interest for sensors and magnetic recording. $5,7,9$ The present work focuses on the micromagnetic behavior of $\mathrm{Co}$ and $\mathrm{Ni}$ wires having radii of less than $10 \mathrm{~nm} .{ }^{10}$ Since extrinsic properties, such as hysteresis and magnetic viscosity, are realized on length scales of the order of $a_{0} / \alpha \approx 7.52 \mathrm{~nm}$, where $a_{0}$ is the Bohr radius and $\alpha$ is Sommerfeld's fine structure constant, ${ }^{11,12}$ thin wires are of particular scientific interest.

Ideal nanowires can be treated as infinite cylinders, and it is possible to discuss the nucleation of reverse domains, that is, essentially the coercivity, in terms of well-known concepts such as coherent rotation and curling. Essentially, coherent or uniform rotation occurs when the wire radius is smaller than $R_{\text {coh }}=3.65 \sqrt{A / \mu_{0} M_{s}^{2}}$, where $A$ is the exchange stiffness and $M_{s}$ is the spontaneous magnetization. Since $R_{\text {coh }}$ is independent of the anisotropy constant $K_{1}$, it is about $10 \mathrm{~nm}$ for typical materials of interest. For $R>R_{\mathrm{coh}}$, the nucleation is realized by magnetization curling. Note that the coherent-rotation and curling modes are delocalized, that is, they extend throughout the wire. ${ }^{12,13}$

Experimental evidence indicates that magnetization reversal is initiated by localized modes. First, it is well known that activation volumes deduced from magnetic viscosity measurements (Sec. IV) are generally much smaller than the wire volumes. This indicates that the reversal starts in a small region of wires. Second, Wegrowe et al., ${ }^{8}$ found that their magnetization data could be fitted to the curling prediction only with the paradoxical assumption that the shape of the inifinite cylinder is like a rugby ball with an aspect ratio of the order of $2: 1$.

The delocalized coherent-rotation and curling modes are exact solutions of the nucleation problem in homogeneous ellipsoids of revolution. ${ }^{12,13}$ They are delocalized because localized modes are more inhomogeneous and, therefore, unfavorable from the point of view of exchange energy. It is conceivable that thermal activation creates a localized nucleus, but examining the energies involved shows that this is a highly unlikely event. In fact, thermal excitations are observed as magnetic viscosity: they slightly reduce the reverse field at which the zero-temperature nucleation mode is realized. The nanomagnetic localization considered in this work reflects the modification of the zero-temperature nucleation mode due to wire imperfections. Since the micromagnetic nucleation problem can be mapped onto a Schrödinger equation, ${ }^{14}$ this micromagnetic localization is akin not only to the localization of nucleation modes in bulk magnets, ${ }^{11,12,15}$ but also to the localization of one-electron wave functions in disordered solids. ${ }^{16}$

Well-known methods to produce magnetic nanowires include vacuum evaporation, electron-beam lithography, and electrodeposition in porous materials. ${ }^{4-9,17,18}$ Only recently has it become possible to produce wires thinner than 20-30 nm. ${ }^{17}$ Here we focus on Co (and Ni) wires deposited in alumina and having radii as small as $5.5 \mathrm{~nm} .{ }^{18}$ The wires were produced by a deposition method very similar to that used in Ref. 17, experimental details are presented in Ref. 18. Very likely, the wires exhibit some length distribution: a recent scanning electron microscope study on Co nanowires deposited in alumina ${ }^{19}$ reports data corresponding to a meansquare deviation of less than $20 \%$. Since our analysis of magnetization data (Sec. IV) covers several orders of magnitude, we will ignore this distribution.

Figure 1 shows the geometry of the wire arrays and-in Fig. 1(a) - the preferred perpendicular magnetization direction as inferred from magnetization measurements. The coercivity, which exceeds $0.2 \mathrm{~T}(2 \mathrm{kOe})$ for $\mathrm{Co}$, is associated with shape anisotropy, but in contrast to previous coherentrotation explanations ${ }^{5-7}$ we consider shape anisotropy realized on a local scale. Figure 1(a) gives a schematic idea of how the magnetization of a localized unit (dark area) reverses. The reason for the localization is that wires may be polycrystalline or textured ${ }^{18}$ rather than single crystalline, ${ }^{8,17}$ and even in single-crystalline wires there are small magnetization perturbances associated with wire-thickness fluctuations, crystalline defects, impurities, and geometrical features at the wire ends.

The aim of this study is to show how small inhomogenities lead to magnetic localization and affect the hysteretic 
(a)
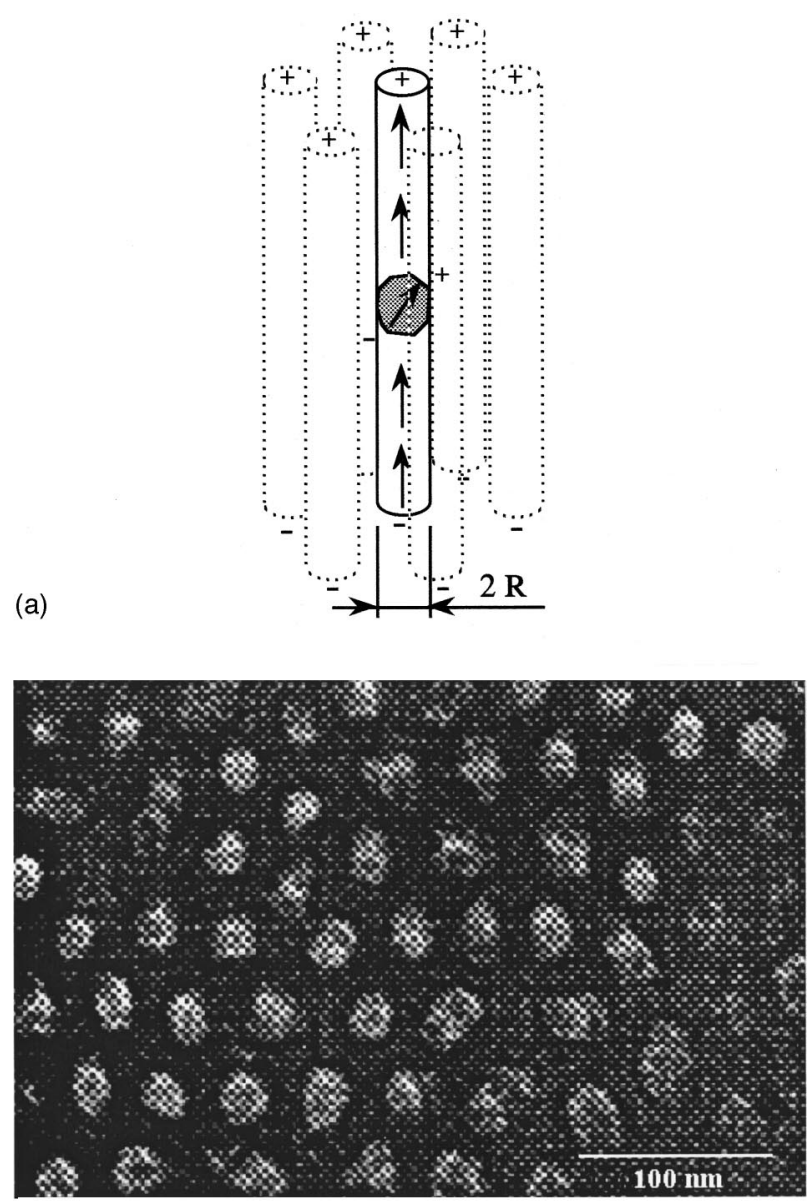

(b)

FIG. 1. Hexagonal array of magnetic nanowires deposited in alumina: (a) schematic picture and (b) Co TEM micrograph (top view). The arrows give the local magnetization direction, whereas the + and - signs denote the corresponding magnetic charges. The magnetic field is parallel to the wires.

behavior. Section II extends the classical random-anisotropy theory of bulk ferromagnets ${ }^{20,21}$ to polycrystalline wires, in Sec. III we calculate the localization length for a nearly homogeneous thin wire, and Sec. IV is devoted to the understanding of magnetic viscosity and coercivity data.

\section{LOCALIZATION IN POLYCRYSTALLINE WIRES}

A morphological feature of fundamental importance in magnetism is the degree of polycrystallinity, or texture. Recent TEM images show that the $\mathrm{Co}$ and $\mathrm{Ni}$ nanowires electrodeposited in alumina pores are polycrystalline. ${ }^{18,22}$ Unlike nearly single-crystalline wires (Sec. III), polycrystalline wires can be interpreted as random-anisotropy ferromagnets. Random-anisotropy effects in bulk magnets have attracted much interest in the past ${ }^{20,21,23,24}$ and reflect the competition between anisotropy and exchange. When the crystallite size $R_{0}$ is smaller than the wall-width parameter $\delta_{0}=\sqrt{A / K_{1}}$ then the exchange interaction ensures a cooperative coupling of neighboring grains, and the behavior of the localization radius $R^{*}$ is governed by the well-known three-dimensional scaling result ${ }^{20}$

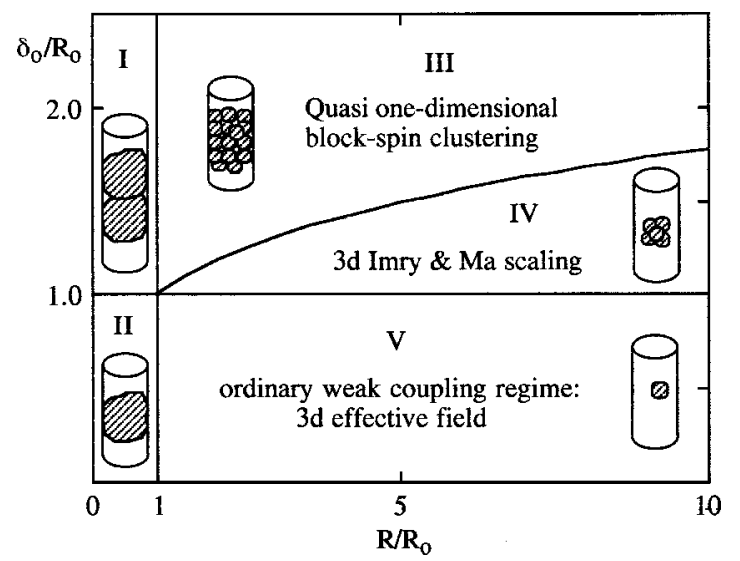

FIG. 2. Magnetic phase diagram for polycrystalline wires. The regions are (I) truly one dimensional and cooperative, (II) truly one-dimensional and noncooperative, (III) quasi-one-dimensional cooperative, (IV) three-dimensional cooperative, and (V) three -dimensional noncooperative. Equations (1), (2), and (3) describe regions (IV), (III), and (I), respectively.

$$
R^{*} \sim \frac{\delta_{0}^{A}}{R_{0}^{3}} .
$$

Essentially, the radius $R^{*}$ of the correlated regions is a domain-wall width calculated self-consistently from the region's average anisotropy. ${ }^{20,21}$ For $\mathrm{Fe}, \mathrm{Co}$, and $\mathrm{Ni}$, the length $\delta_{0}$ is equal to 13,4 , and $26 \mathrm{~nm}$, respectively.

The bulk result Eq. (1) applies to wires whose radius $R$ $>R^{*}>R_{0}$. When the wire radius decreases to $R^{*}$, then there is a transition to a quasi-one-dimensional regime. It is then necessary to distinguish between radial localization, $R_{\perp}^{*}=R$, and longitudinal localization, $R_{\|}^{*} \gg R$. The behavior of the longitudinal localization length $R_{\|}^{*} \gg R$ is onedimensional, but a straightforward extension of the calculations in Refs. 21 and 23 shows that the crystallite size is now renormalized as compared to fictitious one-dimensional chains of nanocrystallites $\left(R=R_{0}\right)$. The quasi-onedimensional localization length is

$$
R^{*} \sim \frac{R^{2 / 3} \delta_{0}^{4 / 3}}{R_{0}},
$$

whereas the localization length for one-dimensional chains of single crystallites scales as

$$
R * \sim \frac{\delta_{0}^{4 / 3}}{R_{0}^{1 / 3}} .
$$

The behavior of polycrystalline wires is summarized in the phase diagram Fig. 2.

The scaling relations Eqs. (1)-(3) provide a qualitative understanding of localization phenomena in polycrystalline wires. The Co nanowires investigated in Ref. 18 are very likely to lie in region III in Fig. 2, but quantitative predictions require the knowledge of the prefactors in the scaling relations, which are of order one. Furthermore, Eqs. (1)-(3) assume that the wire's anisotropy can be mapped onto a single wall-width parameter $\delta_{0}$. This ignores, for example, that Co wires may be a difficult to describe mixture of fcc and hcp crystallites and also neglects the possibility of a 

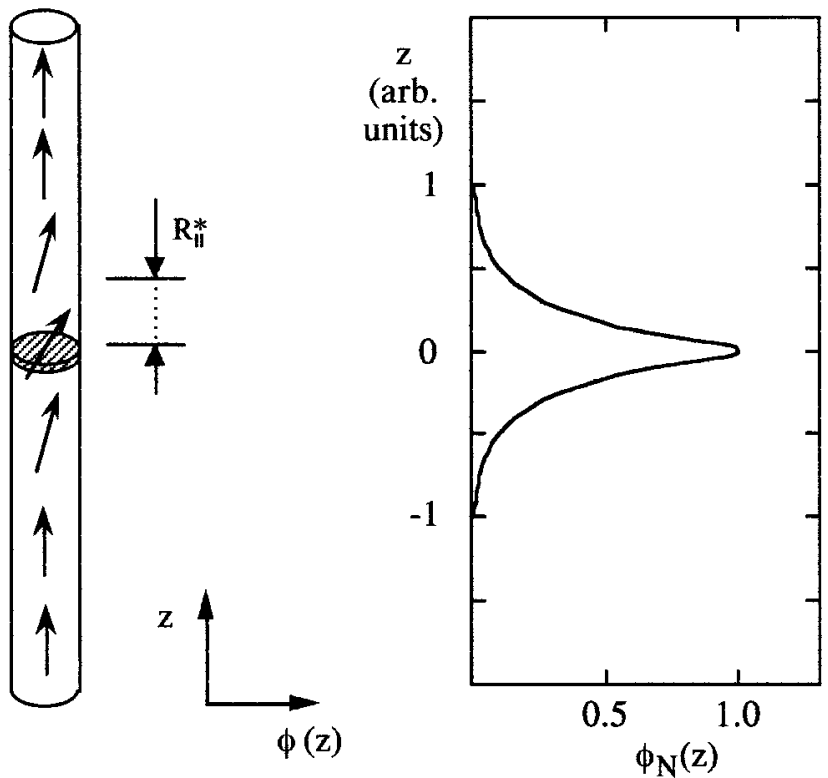

FIG. 3. Nucleation mode localization in a nearly singlecrystalline wire. The length of the perturbed wire region (dashed area) is $L^{\prime}$. After nucleation, the magnetization reversal proceeds by the propagation of two domain walls.

nonrandom or coherent anisotropy along the wire axis. The latter seems to be particularly relevant in $\mathrm{Ni}$ wires. $^{22}$

\section{NEARLY HOMOGENEOUS WIRES}

From the electron-localization analogy ${ }^{16}$ it follows that an arbitrarily weak inhomogenity leads to a localization of the nucleation mode in one dimension. As an exactly solvable special case we consider the limit of very thin wires where the inhomogenity consists in a local reduction of the first anisotropy constant $K_{1}(z)$. In other words, due to soft magnetic impurities or defects, a small part of the wire is slightly softer than the remaining part of the wire. From general magnetic energy expressions (see, e.g., Ref. 12) it follows that

$$
E=\pi R^{2} \int\left[A\left(\frac{\partial \phi}{\partial_{z}}\right)^{2}+\left(K_{1}(z)+\frac{\mu_{0}}{2} M_{s}^{2}+\frac{\mu_{0}}{2} M_{s} H\right) \phi^{2}\right] d z
$$

Here $\phi(z)$ is the angle between the wire axis ( $z$ axis) and the local magnetization and $H=H_{z}$ and $M_{s}$ are the external magnetic field and the spontaneous magnetization, respectively. In the standard way, the nucleation mode $\phi_{N}(z)$ is obtained by minimizing Eq. (4) and solving the resulting eigenvalue problem. Figure 3 shows the nucleation mode for a small, $\delta$-like inhomogenity. The mode decays as $\exp \left(-z / R_{\|}^{*}\right)$, and the localization or decay length is given by

$$
R_{\|}^{*}=\frac{2 A}{\left(K_{1}-K^{\prime}\right) L^{\prime}} .
$$

Here $K_{1}$ and $K^{\prime}<K_{1}$ are the average anisotropy constants in the perturbed and unperturbed regions, respectively, and $L^{\prime}$ is the extension of the perturbation. For example, considering ten atomic layers of hcp cobalt $\left(L^{\prime} \approx 2 \mathrm{~nm}\right)$ whose anisotropy is reduced by about $20 \%$ due to impurities yields the localization length $R_{\|}^{*} \approx 100 \mathrm{~nm}$.
The result Eq. (5) confirms our starting conjecture that any arbitrarily small disorder gives rise to localization and shows that the actual degree of localization (the localization length) is strongly dependent on the wire's microstructure. For zero disorder, the localization length goes to infinity and the reversal degenerates into coherent rotation. It can also be shown that the localization Eq. (5) is accompanied by a reduction of the coercivity. This feature is indeed observed in real wires ${ }^{7,18}$ and bulk magnets. ${ }^{12,15}$ It is closely related to Brown's paradox, ${ }^{12,13}$ which states, somewhat simplifying, that experimental coercivities are smaller than the anisotropy field prediction $H_{c}=2 K_{1} / \mu_{0} M_{s}$.

\section{MAGNETIC VISCOSITY AND COERCIVITY}

Let us now use a simple model to discuss how magnetic localization affects the hysteretic behavior of the wires. A well-known experimental method to investigate the realspace origin of hysteresis are magnetic viscosity measurements, where thermally activated changes in the hysteresis loop are rationalized in terms of a thermal activation volume $V^{*}$. In a standard way, the usually logarithmic ${ }^{4,12,25-27}$ time dependence of extrinsic magnetic properties such as remanence and coercivity is compared with model predictions based on a linear field dependence of the barrier energy, ${ }^{12,27,28}$

$$
E=K V^{*}\left(1-\frac{H}{H_{0}}\right) .
$$

Magnetization reversal in real magnets is usually associated with nonlinear dependences of $E$ on $H$. A widely considered class of nonlinear energy barriers is

$$
E=K V_{0}\left(1-\frac{H}{H_{0}}\right)^{m}
$$

In Eqs. (6) and (7), $K, V_{0}$, and $H_{0}$ are anisotropy, volume, and field parameters, respectively, whose detailed definition depends on the underlying model. The exponent $m$ varies between 1.5 to 2 for a variety of pinning and nucleation models. ${ }^{25-27}$ Equation (7) corresponds to rectangular hysteresis loops, but due to thermal activation the finitetemperature coercivity is $H_{c}<H_{0}{ }^{29}$

Let us make the model assumption that magnetization reversal is initiated by the locally coherent rotation of a localized cooperative Stoner-Wohlfarth unit $(m=2)$ in a combined magnetostatic ${ }^{18}$ and exchange mean field, as implied in Fig. 1(a). The thermal activation volume is then given by (compare Ref. 27).

$$
V^{*}=2 \sqrt{V_{0}} \sqrt{k_{B} T \ln \left(\Gamma_{0} t\right) / K} .
$$

Due to the nonlinearity of the model, $m=2$ in Eq. (7), $V^{*}$ must be distinguished very clearly from the physical volume $V_{0}$. The corresponding finite-temperature coercivity is $H_{c}$ $=H_{0}\left(1-\sqrt{k_{B} T \ln \left(\Gamma_{0} t\right) / V_{0} K}\right){ }^{12,23,25}$ Since the factor $k_{B} T \ln \left(\Gamma_{0} t\right)$ is of the order of $25 k_{B} T$ for laboratory scale experiments, the two equations for $V^{*}$ and $H_{c}$ provide a basis for the discussion of experimental data.

Figure 4 compares the $V^{*}$ and $H_{c}$ predictions with experimental data; the fitting parameters are $V_{0}$ and $K$. Since $V^{*}$ is proportional to $\sqrt{V_{0}}$ and $V_{0} \approx \pi R^{2} L$, Eq. (8) predicts a square-root dependence of $V^{*}$ on the wire length $L$. Figure 

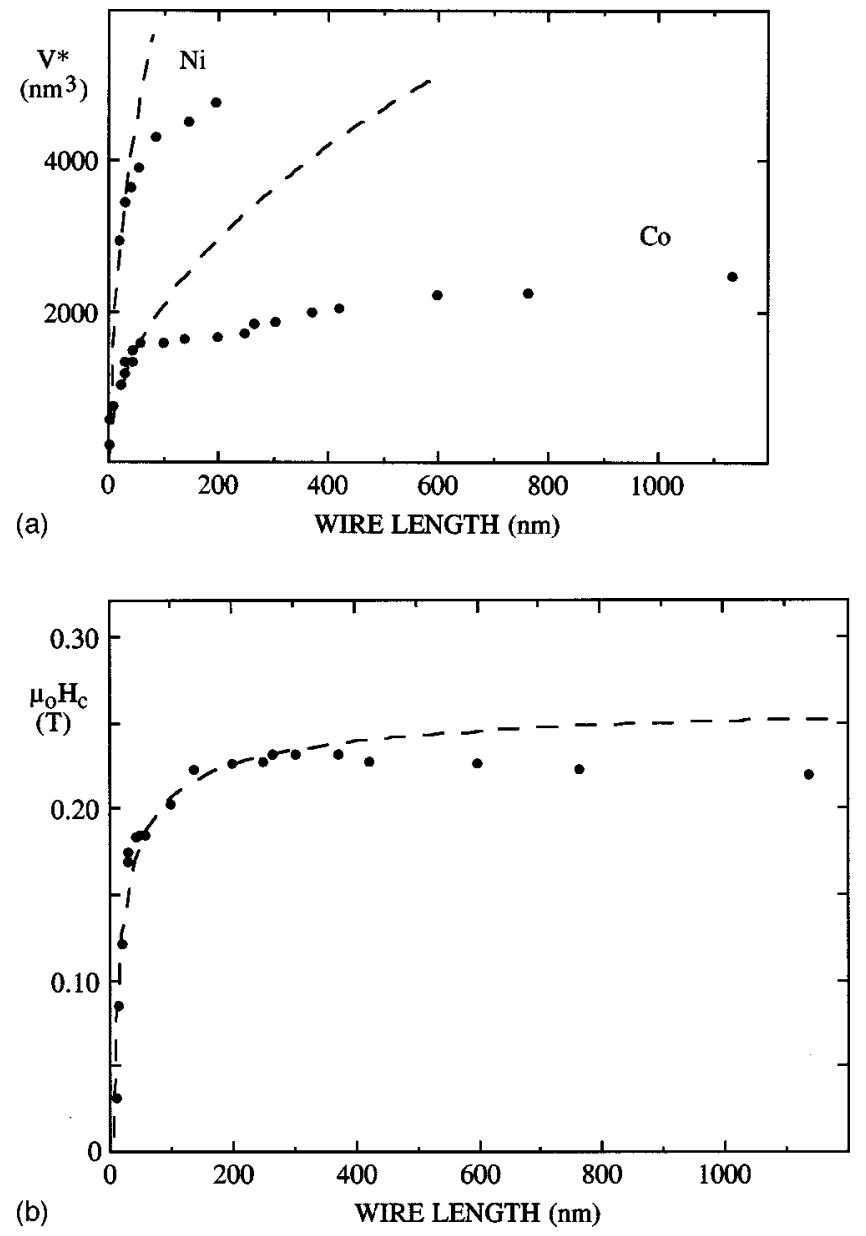

FIG. 4. Wire-length dependence of hysteretic properties: (a) thermal activation volume of $\mathrm{Co}$ and $\mathrm{Ni}$ nanowires and (b) coercivity of Co nanowires. The experimental data refer to wires whose lengths have been modified by changing the deposition conditions (time and voltage). The wire radii are 5.5 and $10 \mathrm{~nm}$ for Co (Ref. 17) and Ni (Ref. 20), respectively. The dashed lines are theoretical predictions based on the assumption that the physical activation volume is proportional to the wire length.

4(a) shows that the square-root dependence (dashed lines) works fairly well for short wires but breaks down above a critical length. This confirms the theoretical predictions: when the wire length exceeds a critical value of the order of $R_{\|}^{*}$ then the physical volume $V_{0}$ (and the activation volume $V^{*}$ ) remain largely unchanged. Figure 4(b) compares the coercivity prediction with the experimental dependence of the coercivity of Co on the wire length $L$. For sufficiently large physical switching volumes, that is, for not-too-short wires, the Stoner-Wohlfarth prediction is only weakly volume dependent $\left(H_{c} \approx H_{0}\right)$, so that the numerical disagreement between Stoner-Wohlfarth theory and experiment is small. The slight deterioration of the coercivity of very long wires is reminiscent of the finite-size behavior of bulk and thin-film magnets, and means ${ }^{12}$ that large magnets are more likely to contain pronounced inhomogenities harmful to coercivity.

As compared to the scaling approach (Fig. 2) and to the exact model result Eq. (5), Fig. 4 provides a qualitatively correct explanation of the complicated interplay of wire morphology, real-space magnetization behavior, magnetic viscosity, and coercivity. However, the quantitative agreement is less comprehensive. In the case of Co, where most data points are available, the not unreasonable value $K$ $=0.425 \mathrm{MJ} / \mathrm{m}^{3}$ provides a simultaneous fitting of Figs. 4(a) and 4(b) only if one chooses $V_{0}=1.54 R^{2} L$ rather than $V_{0}$ $=\pi R^{2} L$. Taking into account the transparent simplicity of the model Eq. (7), this inaccuracy is neither surprising nor weighty. One factor is that the magnetostatic shape anisotropy contribution to the anisotropy, which originates from the poles shown in Fig. 1(a), is parametrized in terms of $K$ rather than calculated.

\section{CONCLUSIONS}

By examining various systems we have shown that nanomagnetic localization in real nanowires is a common phenomenon, caused by structural features and largely responsible for the observed hysteretic behavior. This explains, for example, the puzzling rugby-ball-like behavior of the nanowires investigated in Ref. 8 and the smallness of magnetic-viscosity activation volumes in long wires. Polycrystalline wires are discussed in terms of a randomanisotropy hierarchy, and scaling relations are derived for the different regimes. For a nearly ideal model nanowire an exact localization expression has been obtained, showing that the localization length increases with decreasing wire inhomogenity.

\section{ACKNOWLEDGMENTS}

Thanks are due to Dr. S. Wirth for stimulating discussions. This research was supported by DOE, DOD, and NSF under Grant No. DMR0623992, IBM, NSIC, and CMRA.
*Corresponding author. Email address: rskomski@unlserve.unl.edu

${ }^{1}$ E. Ising, Z. Phys. 31, 253 (1925).

${ }^{2}$ L. J. de Jongh and A. R. Miedema, Adv. Phys. 23, 1 (1974).

${ }^{3}$ J. Shen, R. Skomski, M. Klaua, H. Jenniches, S. S. Manoharan, and J. Kirschner, Phys. Rev. B 56, 2340 (1997).

${ }^{4}$ W. Wernsdorfer, B. Doudin, D. Mailly, K. Hasselbach, A. Benoit, J. Meier, J.-Ph. Ansermet, and B. Barbara, Phys. Rev. Lett. 77, 1873 (1996).

${ }^{5}$ M. Masuda, Sh. Shiomi, and M. Shiraki, Jpn. J. Appl. Phys., Part 1 26, 1680 (1987)

${ }^{6}$ T. M. Whitney, J. S. Jiang, P. C. Searson, and C. L. Chien, Science 261, 1318 (1993).
${ }^{7}$ G. Zangari and D. N. Lambeth, IEEE Trans. Magn. 33, 3010 (1997).

${ }^{8}$ J.-E. Wegrowe, D. Kelly, A. Fromck, S. E. Gilbert, and J.-Ph. Ansermet, Phys. Rev. Lett. 82, 3681 (1999).

${ }^{9}$ J.-E. Wegrowe, D. Kelly, Y. Jaccard, Ph. Guittienne, and J.-Ph. Ansermet, Europhys. Lett. 45, 626 (1999).

${ }^{10}$ In terms of the definition introduced by W. F. Brown, Micromagnetics (Wiley-Interscience, New York, 1963), the present approach is micromagnetic (continuum-theoretical), because electronic-structure effects enter the theory as atomic parameters (see, e.g., Ref. 12). 
${ }^{11}$ R. Skomski, H.-P. Oepen, and J. Kirschner, Phys. Rev. B 58, 3223 (1998).

${ }^{12}$ R. Skomski and J. M. D. Coey, Permanent Magnetism (Institute of Physics, Bristol, 1999).

${ }^{13}$ A. Aharoni, Introduction to the Theory of Ferromagnetism (Oxford University Press, Oxford, 1996).

${ }^{14}$ R. Skomski and J. M. D. Coey, Phys. Rev. B 48, 15812 (1993).

${ }^{15}$ R. Skomski, J. Appl. Phys. 83, 6503 (1998).

${ }^{16}$ P. W. Anderson, Phys. Rev. 109, 1492 (1958).

${ }^{17}$ F.-Y. Li and R. M. Metzger, J. Appl. Phys. 81, 3806 (1997).

${ }^{18}$ H. Zeng, M. Zheng, R. Skomski, D. J. Sellmyer, Y. Liu, L. Menon, and S. Bandyopadhyay, J. Appl. Phys. 87, 4718 (2000).

${ }^{19}$ R. M. Metzger, V. V. Konovalov, M. Sun, T. Xu, G. Zangari, B. $\mathrm{Xu}, \mathrm{M}$. Benakli, and W. D. Doyle, IEEE Trans. Magn. 36, 30 (2000).
${ }^{20}$ R. Alben, M. C. Chi, and J. J. Becker, J. Appl. Phys. 49, 1653 (1978).

${ }^{21}$ E. M. Chudnovsky, W. M. Saslow, and R. A. Serota, Phys. Rev. B 33, 251 (1986).

${ }^{22}$ M. Zheng, R. Skomski, Y. Liu, and D. J. Sellmyer (unpublished).

${ }^{23}$ Y. Imry and Sh.-K. Ma, Phys. Rev. Lett. 35, 1399 (1975).

${ }^{24}$ D. J. Sellmyer and S. Nafis, Phys. Rev. Lett. 57, 1173 (1986).

${ }^{25}$ E. Kneller, Ferromagnetisms (Springer, Berlin, 1962).

${ }^{26}$ P. Gaunt, J. Appl. Phys. 59, 4129 (1986).

${ }^{27}$ R. Skomski, R. D. Kirby, and D. J. Sellmyer, J. Appl. Phys. 85, 5069 (1999).

${ }^{28}$ D. J. Sellmyer, M. Yu, R. A. Thomas, Y. Liu, and R. D. Kirby, Phys. Low-Dimens. Semicond. Struct. 1-2, 155 (1998).

${ }^{29}$ For simplicity we ignore the intrinsic temperature dependence of the parameters $K$ and $H_{0}$. Nonrectangular hysteresis loops indicate a distribution of energy barriers $E$ (Ref. 12). 